\title{
Cognitive Computing Track Chairs' Welcome
}

Cognitive Computing has received increasing attention from academia and industries as it brings cognitive science and computing together for the development of new computational platforms, infrastructures, systems and algorithms. Artificial intelligence and computational intelligence are key elements to succeed in cognitive computing.

The Cognitive Computing track was successfully organized at WWW2017. This year, WWW2018 continues this track with a focus on all applications. Particularly, applications in healthcare, environment, education, sustainability, smart cities and food science are among the important global challenges in 21st Century.

We invited submissions from academia as well as industry and government sectors. All contributions focused on the use of cognitive computing. It is our great pleasure to welcome you to the WWW 2018 Cognitive Computing Track. We secured an amazing number of novel research works from all around the world covering a broad range of topics including but not limited to:

- Cognitive Computing and WWW topics

- Cognitive Computing Technologies

- Cognitive Computing / Industry Applications as applied in WWW

We evaluated them regarding relevance, quality, geographical distribution of contributors, and novelty, selecting 17 full papers and 6 short papers for oral presentation and inclusion to the proceedings. We also took in account the coverage of the different areas related to WWW as well as the potential audience, to schedule them in two consecutive days with the minimal audience interest overlap.

The morning of the third day includes the following six talks:

- AI Cognition in Searching for Relevant Knowledge from Scholarly Big Data, Using a Multilayer Perceptron and Recurrent Convolutional Neural Network Model

Authors: Saeed-Ul Hassan, Iqra Safder and Naif Aljohani

- A framework for human-in-the-loop monitoring of concept-drift detection in event log stream

Authors: Sylvio Barbon Junior, Gabriel Marques Tavares, Victor Guilherme Turrisi Da Costa, Paolo Ceravolo and Ernesto Damiani

- Analyzing and Predicting Emoji Usages in Social Media

Authors: Peijun Zhao, Jia Jia, Yongsheng An, Jie Liang, Lexing Xie and Jiebo Luo

- Automatic Hierarchical Table of Contents Generation for Educational Videos

Authors: Debabrata Mahapatra, Ragunathan Mariappan and Vaibhav Rajan

- The Grass is Greener on the Other Side: Understanding the Effects of Green Spaces on Twitter User Sentiments

Authors: Kwan Hui Lim, Kate E. Lee, Dave Kendal, Lida Rashidi, Elham Naghizade, Stephan Winter and Maria Vasardani

- Lifecycle-Based Event Detection from Microblogs

Authors: Lin Mu, Peiquan Jin, Lizhou Zheng, En-Hong Chen and Lihua Yue 
The afternoon of the third day includes the following six talks

- Human-Guided Flood Mapping: From Experts to the Crowd Authors: Jiongqian Liang, Peter Jacobs and Srinivasan Parthasarathy

- Human-level multiple choice question guessing without domain knowledge: Machinelearning of framing effects.

Authors: Patrick Watson, Tengfei Ma, Ravi Tejwani, Jaewook Ahn, Maria Chang and Sharad Sundararajan

- A Context-Aware NeuRetrieval Model for Human-Computer Conversation Authors: Rui Yan and Dongyan Zhao

- How to improve the answering effectiveness in Pay-for-Knowledge Community: An exploratory application of Intelligent QA System

Authors: Yihang Cheng, Xi Zhang, Hao Wang and Shan Jiang

- Region-wise Ranking of Sports Players based on Link Fusion

Authors: Ali Daud, Akbar Hussain, Rabeeh Ayaz Abbasi, Naif Radi Aljohani, Tehmina Amjad and Hassan Dawood

- Measuring the Impact of Topic Drift in Scholarly Networks

Authors: Tehmina Amjad, Ali Daud and Min Song

The morning of the fourth day includes the following six talks:

- PersuAIDE! An Adaptive Persuasive Text Generation System for Fashion Domain

Authors: Vitobha Munigala, Abhijit Mishra, Srikanth Govindaraj Tamilselvam, Shreya Khare, Riddhiman Dasgupta and Anush Sankaran

- When E-commerce Meets Social Media: Identifying Business on WeChat Moment Using Bilateral-Attention LSTM

Authors: Tianlang Chen, Yuxiao Chen, Han Guo and Jiebo Luo

- Disease Tracking in GCC Region Using Arabic Language Tweets

Authors: Muhammad Usman Ilyas and Jalal S. Alowibdi

- Learning Procedures from Text: Codifying How-to Procedures in Deep Neural Networks

Authors: Hogun Park and Hamid Reza Motahari Nezhad

- Pain Prediction in Humans using Human Brain Activity Data

Authors: Mustansar Ali Ghazanfar, Zara Mansoor, Syed M Anwer, Ahmed S. Alfakeeh and Khaled H. Alyoubi

- Making the Most Cost-effective Decision in Online Paid Q\&A Community: An Expert Recommender System with Motivation Modeling and Knowledge Pricing

Authors: Yunhao Zheng, Xi Zhang and Yuting Xiao

The afternoon of the fourth day includes the following five talks:

- Discovering Connotations as Labels for Weakly Supervised Image-Sentence Data 
Authors: Aditya Mogadala, Bhargav Kanuparthi, Achim Rettinger and York Sure-Vetter

- $\quad$ Perceiving Commerial Activeness Over Satellite Images

Authors: Zhiyuan He, Su Yang, Weishan Zhang and Jiulong Zhang

- Netizen-Style Commenting on Fashion Photos: Dataset and Diversity Measures

Authors: Wen Hua Lin, Kuan-Ting Chen, Hung Yueh Chiang and Winston Hsu

- $\quad$ Learning the Chinese Sentence Representation with LSTM Autoencoder

Authors: Mu-Yen Chen, Tien-Chi Huang, Yu Shu, Chia-Chen Chen, Tsung-Che Hsieh and Neil Y. Yen

- Activity-Based Mobility Profiling: A Purely Temporal Modeling Approach

Authors: Shreya Ghosh, Soumya K Ghosh, Rahul Deb Das and Stephan Winter

The call for tutorials attracted submissions from United States, Europe, Asia, Africa and South America. Review and acceptance statistics are as follows:

\begin{tabular}{|l|c|c|c|}
\hline Submission Type & Reviewed & Accepted & \\
\hline Full Technical Papers & 52 & 17 & $30 \%$ \\
\hline Short Poster Presentations & & 6 & $12 \%$ \\
\hline
\end{tabular}

We believe that the program provides a good balance between several trending topics such as deep learning, social media analysis, graph mining, crowdsourcing, knowledge databases, mobile data, etc. Hence we hope that you will find the tutorial program interesting, providing you with a valuable opportunity to learn and share ideas with other researchers and practitioners from institutions around the world.

$\begin{array}{ll}\text { Miltiadis D. Lytras } & \text { Jiebo Luo } \\ \text { Lead Track Co-Chair } & \text { Track Co-Chair } \\ \text { The American College of Greece } & \text { University of Rochester } \\ \text { Naif Radi Aljohani } & \text { Amir Hussain } \\ \text { Track Co-Chair } & \text { Track Co-Chair } \\ \text { King Abdulaziz University } & \text { University of Stirling } \\ \text { Jacky Xi Zhang } & \\ \text { Track Co-Chair } & \\ \text { Tianjin University } & \end{array}$




\section{Cognitive Computing Track Organization}

Workshop/Track Chairs: Miltiadis D. Lytras (The American College of Greece, Greece)

Naif Radi Aljohani (King Abdulaziz University, Saudi Arabia)

Amir Hussain (University of Stirling, United Kingdom)

Jiebo Luo (University of Rochester, USA)

Jacky Xi Zhang (Tianjin University, P. R. China)

Program Committee and Marie Helene Abel (Université de technologie de Compiègne, France)

Reviewers: Wadee Alhalabi (Effat University, Saudi Arabia)

Sachi Arafat (University of Glasgow, Scotland, UK)

Nicola Capuano (University of Salerno, Italy)

Ernesto Damiani (EBTIC/Khalifa University, Abu Dhabi, UAE)

Ernesto Davadzo (University of Salerno, Italy)

Ramzi Haraty (Lebanese American University, Lebanon)

Peiquan Jin (University of Science and Technology, China)

Eugenijus Kurilovas (Vilnius University Institute of Mathematics and

Informatics, Lithuania, Lithuania)

Inma Rodríguez-Ardura (Universitat Oberta de Catalunya, Spain)

Akila Sarirete (Effat University, Saudi Arabia)

Chien-wen Shen (National Central University, Taiwan)

Miguel Ángel Sicilia (University of Alcala, Spain)

Marco Spruit (Utrecht University, The Netherlands)

Marco Temperini (SAPIENZA Università di Roma, Italy)

Miguel Torres (Instituto Politécnico Nacional, Mexico)

Yves Wauletet (KU Leuven, Belgium)

Jim Wu (National Taiwan Normal University, Taiwan)

Cornelio Yanez-Marquez (Instituto Politécnico Nacional, Mexico)

Leyla Zhuhadar (Western Kentucky University, United States) 